\title{
Sorption of Fluorinated Azo Dyes Containing Two Sulfonate Groups by a Nylon 6 Film $^{* 1}$
}

\author{
Kunihiro Hamada, Ken-ichi Oshiki and Hirofusa Shirai \\ Faculty of Textile Science and Technology, Shinshu University \\ 3-15-1 Tokida, Ueda, Nagano 386-8567, Japan
}

\begin{abstract}
Derivatives of disodium 1-phenylazo-2-hydroxy-3,6-naphthalenedisulfonate, some of which contain a trifluoromethyl or methyl group, were prepared and their sorption behavior by a nylon 6 film at pH3 was investigated. The dye uptake by the nylon 6 film increased linearly with sorption time. The slopes of the linear plots varied depending on the dye structure: the kind and number of the substituents in the dyes affected the slopes. The analysis of the sorption isotherms based on a dual sorption mechanism, which consists of partition and Langmuir type sorption, gave the partition coefficients $\left(K_{\mathrm{P}}\right)$ and the number of binding sites $(n)$ and intrinsic binding constants $\left(K_{\mathrm{L}}\right)$ for the Langmuir type sorption. The introduction of the trifluoromethyl or methyl group into the dye molecules hardly influenced $K_{\mathrm{P}}$ and $n$, while it changed the $K_{\mathrm{L}}$ values significantly. Furthermore, thermodynamic parameters, enthalpy change and entropy change, for the Langmuir type sorption were greatly affected by the replacement of the substituents, suggesting that the hydrophobic interaction should be concerned with the Langmuir type sorption.
\end{abstract}

\section{Introduction}

Anionic groups of acid dyes play an important role in their sorption by a nylon 6 film [1]. This is due to Langmuir type sorption of the acid dyes by the nylon 6 film: the protonated amino end groups in the nylon behave as sites for the anionic groups of the dyes. As dyes containing two anionic groups interact with two cationic binding sites in the nylon 6 film, their uptake becomes half of those for dyes having a single anionic group.

On the other hand, the effects of functional groups different from the anionic groups on the sorption are of interest. We have investigated the effects of fluorine atoms on the sorption of acid dyes containing a trifluoromethyl group by a nylon 6 film [2-4] and a silk

*1Studies on the Sorption of Azo Dyes Containing Fluorine Atoms by a Nylon 6 Film (Part 3) fiber [5]. As a result, the introduction of the fluorine atoms made enthalpy change and entropy change for the Langmuir type sorption more exothermic and less entropic, respectively, in the case of the nylon 6 film. Furthermore, the position of the trifluoromethyl group affected the thermodynamic parameters. Savarino et al. investigated the effects of substituents on dyeing properties of a polyamide fiber using derivatives of 1-phenylazo-2-alkylamido-5-hydroxy-4-benzoic acid $[6,7]$ and derivatives of 1-(2-alkylamido-4hydroxyphenylazo)-4-benzenesulfonic acid [8]. They concluded that the dyes containing longer alkyl chains showed higher dye uptake due to their hydrophobicity.

In the present study, the sorption of fluorinated azo dyes containing two sulfonate groups by a nylon 6 film was investigated. The sorption isotherms obtained were analyzed by considering a dual sorption mechanism. The effects of the trifluoromethyl and sulfonate groups are discussed. 


\section{Experimental}

\subsection{Dyes}

Seven azo dyes, disodium 1-phenylazo-2-hydroxy-3,6naphthalenedisulfonate (AR), disodium 1-(2-trifluoromethylphenylazo) -2-hydroxy-3,6-naphthalenedisulfonate (o-FTR), 1-(3-trifluoromethylphenylazo)-2hydroxy-3,6-naphthalenedisulfonate (m-FTR), 1-(4trifluoromethylphenylazo)-2-hydroxy-3,6-naphthalenedisulfonate (p-FTR), 1-(2-methylphenylazo)-2hydroxy-3,6-naphthalenedisulfonate (o-TR), 1-(3methyl-phenylazo) -2-hydroxy-3,6-naphthalenedisulfonate (m-TR), and 1-(4-methylphenylazo)-2hydroxy-3,6-naphthalenedisulfonate ( $\mathrm{p}$-TR) were used. The structure of these dyes is shown in Fig. 1.
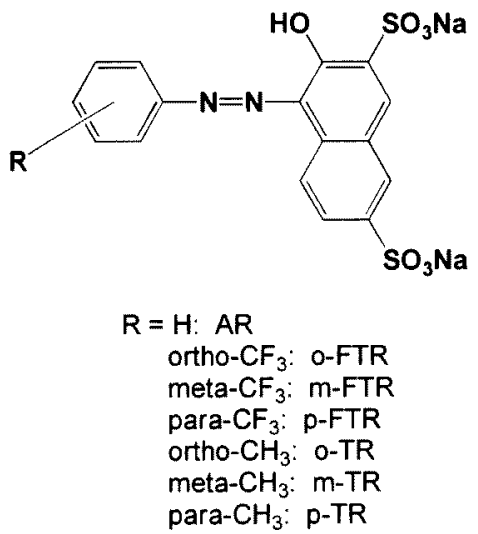

Fig. 1 Dyes used.

AR (Ponceau G) was purchased from Tokyo Kasei Co. (Tokyo, Japan) and purified by repeated recrystallization from aqueous ethanol [9]. The syntheses of $m-F T R, m-T R$, and $p-T R$ are reported in previous papers [10-12]. o-FTR, p-FTR, and o-TR were synthesized by coupling the corresponding diazotized aniline derivatives (2-trifluoromethylaniline, 4-trifluoromethylaniline, and 2-toluidine) with $\mathrm{R}$ acid (2-naphthol-3,6-disulfonic acid) in an alkaline condition. The dyes thus obtained were purified by repeated salting-out with $\mathrm{NaCl}$ and reprecipitating the aqueous solutions with acetone. The purity of the dyes was confirmed by an elemental analysis.

\subsection{Nylon 6 Film}

Biaxially drawn nylon 6 film was kindly supplied by Unitika Co., whose thickness was $15 \mu \mathrm{m}$. The amino end group content was determined as $3.53 \times 10^{-5}$ equivalent per $\mathrm{g}$ of nylon by the titration of a $\mathrm{m}$-cresol/methanol solution of the nylon 6 with an aqueous methanol solution of hydrochloric acid. This accurate titration showed that the amino end group content $\left(4.65 \times 10^{-5}\right.$ eq $\mathrm{g}^{-1}$ ) described in the previous papers $[3,4]$ was not correct. The nylon 6 film was pretreated in boiling water for $90 \mathrm{~min}$, immersed in distilled water for 1 day, soaked in aqueous hydrochloric acid of $\mathrm{pH} 3$ for 1 day, and dried in a desiccator with silica gel.

\subsection{Dye Sorption by Nylon 6 Film}

The time dependence of dye sorption was determined by soaking $20 \mathrm{mg}$ of the pretreated nylon 6 film in the aqueous dye solution $(50 \mathrm{~mL})$ conditioned at $\mathrm{pH} 3$ by a suitable amount of hydrochloric acid. After a desired time, the film was removed from the dye bath, rinsed in cold water, blotted with filter papers to remove the remaining dye solution on the film surface, and dried in a desiccator with silica gel. The dye sorbed by the film was extracted with $25 \mathrm{~mL}$ of $25 \%$ aqueous pyridine solution and its concentration was determined spectrophotometrically using Shimadzu UV.3100 spectrophotometer. The amount of dye sorbed by the nylon 6 film was calculated from the dye concentration of the extracted solution.

Sorption isotherms were determined in the same manner as described above. In this case, the sorption time was 1 day.

\section{Results and Discussion}

\subsection{Time Dependence of Dye Sorption}

The time dependence of the amount of m-FTR sorbed by the nylon 6 film is shown in Fig. 2 together with that for sodium 1-(3-trifluoromethylphenylazo) -2-hydroxy6-naphthalenesulfonate (m-FTS) [4], where $r$ denotes the amount (mol) of dye sorbed by $1 \mathrm{~g}$ of the nylon 6 film and the initial dye concentration expresses the concentration of the dye bath before the dye is sorbed by the nylon 6 film. If the plots of $r$ against time are assumed to be linear, the slopes show the degree of the time dependence. The slopes were determined by means of the linear least-squares method, and are given in Table 1 together with the corresponding data for derivatives of sodium 1-phenylazo-2-hydroxy-6. naphthalenesulfonate.

The slopes were diminished by increasing number of 


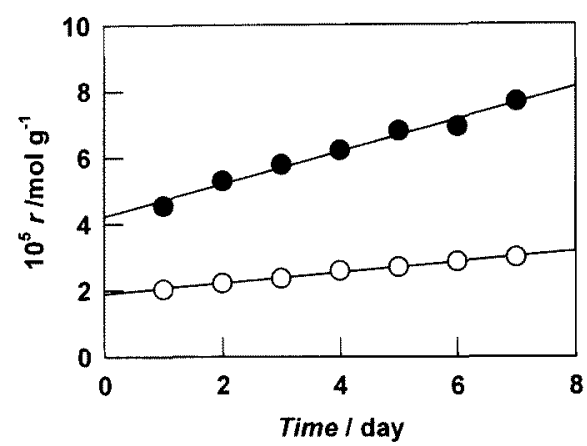

Fig. 2 Time dependence of the amount of dye sorbed by the nylon 6 film at $353 \mathrm{~K}$ for $\mathrm{m}-\mathrm{FTR}(\mathrm{O})$ and $\mathrm{m}$-FTS $(-)$. Initial dye concentration is $1 \times 10^{-4} \mathrm{~mol} \mathrm{dm}^{-3}$.

Table 1 Slopes $\left(\mathrm{mol} \mathrm{g}^{-1} \mathrm{day}^{-1}\right)$ in the Plots of $r$ against Time.

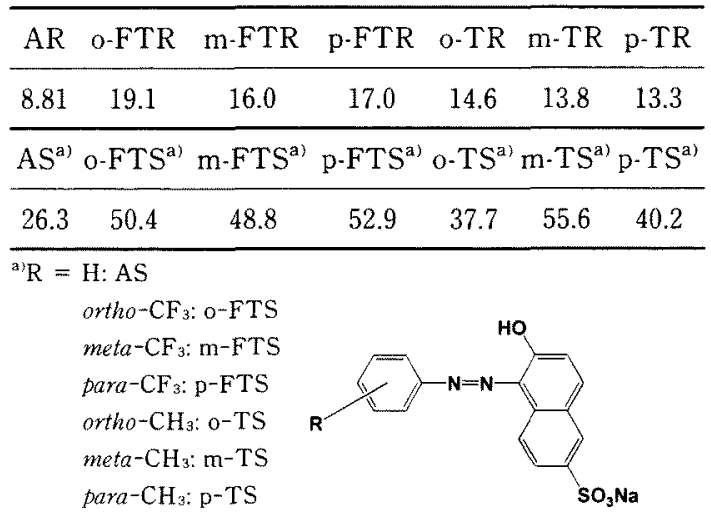

sulfonate groups, suggesting that the anionic groups play an important role in the time dependence of the dye sorption. For both series of dyes containing one and two sulfonate groups, the introduction of trifluoromethyl and methyl group into the dye molecules made the slopes larger by a factor of about 2 . This suggests that the substituents other than the sulfonate group also influence the time dependence of the dye sorption. As was pointed out in our previous papers [3,4], Langmuir type sorption is independent of sorption time, while partition is strongly dependent. Therefore, the difference of the time dependence in the substituents is probably due to the discrepancy of the effects on the partition type sorption. If a simple partition mechanism is dominant in the sorption through nonelectrostatic interaction, the diminishing effect of the sulfonate and the enhancing effect of trifluoromethyl and methyl are reasonable. The positional effects of the substituents were observed for the dyes containing one methyl and one sulfonate group. This reason is not clear, although the electron-releasing and electron-attracting properties of the substituents are presumably concerned with the positional effects.

\subsection{Sorption Isotherms}

As described in the previous section, the previous papers $[4,5]$ demonstrate that the Langmuir type sorption does not depend on time, whereas the partition type sorption does. Therefore, the sorption isotherms were determined on the basis of the dye sorption for 1 day. The sorption isotherm thus determined for $\mathrm{m} \cdot \mathrm{FTR}$ at $353 \mathrm{~K}$ is shown in Fig. 3 together with that for $\mathrm{m}$-FTS [4]. Here, $C_{\text {free }}$ is the concentration of free dyes in the bath. The amounts of dye sorbed for the dyes containing two sulfonate groups were reduced to one-half that for the dyes having one sulfonate, whose reason will be discussed in the later section.

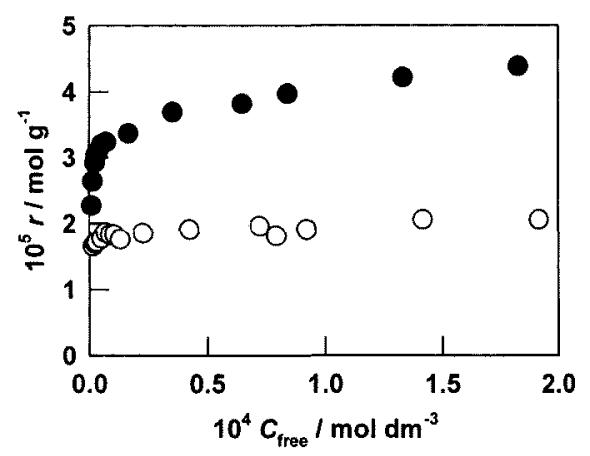

Fig. 3 Sorption isotherms of m-FTR $(O)$ and m.FTS (O) for the nylon 6 film at $353 \mathrm{~K}$.

The sorption isotherms were analyzed by means of the following bimodal sorption equation $[1,3,4]$ :

$$
r=K_{\mathrm{P}} \cdot C_{\text {free }}+\frac{n \cdot K_{\mathrm{L}} \cdot C_{\text {free }}}{1+K_{\mathrm{L}} \cdot C_{\text {free }}}
$$

where $K_{\mathrm{P}}$ is the partition coefficient, $n$ the number of binding sites for the Langmuir type sorption, and $K_{\mathrm{L}}$ the intrinsic binding constant for the Langmuir type sorption. The first and second term in the right-hand side of Eq. 1 express the dye uptake by the partition and Langmuir type sorption, respectively. The partition 
type sorption term may contain an activity effect on the free dye in the higher concentration region.

The $K_{\mathrm{P}}, n$, and $K_{\mathrm{L}}$ values were determined in the same manner as described in our previous paper [3] and are given in Tables 2,3 , and 4 , respectively. The experimental data were exactly fitted by a curve drawn using the sorption parameters thus obtained (Fig. 4), suggesting the validity of the dual sorption mechanism.

The partition coefficients, $K_{\mathrm{P}}$, are dependent on sorption time, as was pointed out in the previous reports $[3,4]$, so that the values for each dye can not be simply

Table 2 Partition Coefficients, $10^{2} K_{\mathrm{P}}\left(\mathrm{dm}^{3} \mathrm{~g}^{-1}\right)$

\begin{tabular}{lcccc}
\hline & $333 \mathrm{~K}$ & $343 \mathrm{~K}$ & $353 \mathrm{~K}$ & $363 \mathrm{~K}$ \\
\hline AR & 0.73 & 0.76 & 1.02 & 1.41 \\
o-FTR & 1.33 & 1.22 & 1.75 & 2.00 \\
m-FTR & 1.17 & 1.19 & 1.45 & 1.87 \\
p-FTR & 1.53 & 1.59 & 1.47 & 1.67 \\
o-TR & 1.06 & 1.09 & 1.30 & 1.75 \\
m-TR & 1.18 & 1.18 & 1.81 & 2.20 \\
p-TR & 0.73 & 1.08 & 1.38 & 2.19 \\
\hline
\end{tabular}

Table 3 Number of Binding Sites, $10^{5} n\left(\mathrm{~mol} \mathrm{~g}^{-1}\right)$

\begin{tabular}{lcccc}
\hline & $333 \mathrm{~K}$ & $343 \mathrm{~K}$ & $353 \mathrm{~K}$ & $363 \mathrm{~K}$ \\
\hline AR & 1.54 & 1.53 & 1.52 & 1.60 \\
o-FTR & 1.66 & 1.71 & 1.72 & 1.76 \\
m.FTR & 1.76 & 1.77 & 1.83 & 1.89 \\
p.FTR & 1.67 & 1.70 & 1.70 & 1.75 \\
o-TR & 1.73 & 1.76 & 1.76 & 1.77 \\
m-TR & 1.94 & 1.93 & 1.84 & 1.90 \\
p-TR & 1.77 & 1.77 & 1.74 & 1.81 \\
\hline
\end{tabular}

Table 4 Intrinsic Binding Constants, $10^{-5} K_{\mathrm{L}}$ $\left(\mathrm{dm}^{3} \mathrm{~mol}^{-1}\right)$

\begin{tabular}{lcccc}
\hline & $333 \mathrm{~K}$ & $343 \mathrm{~K}$ & $353 \mathrm{~K}$ & $363 \mathrm{~K}$ \\
\hline AR & 39 & 35 & 29 & 24 \\
o.FTR & 130 & 105 & 83 & 62 \\
m.FTR & 151 & 96 & 67 & 49 \\
p-FTR & 130 & 92 & 74 & 56 \\
o-TR & 30 & 31 & 32 & 32 \\
m-TR & 65 & 58 & 54 & 54 \\
p-TR & 56 & 52 & 55 & 54 \\
\hline
\end{tabular}

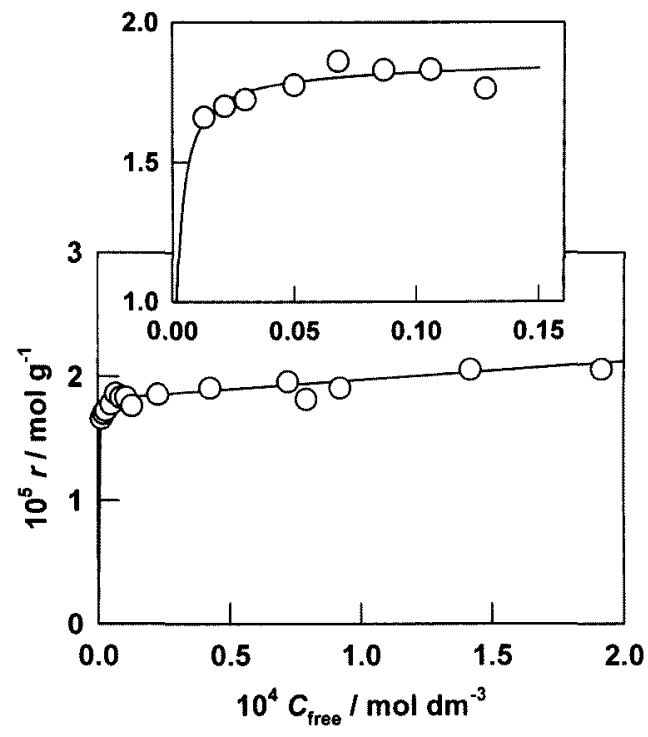

Fig. 4 Sorption isotherm of m-FTR calculated using the sorption parameters based on the dual sorption mechanism.

compared. However, the results for the time dependence described in the previous section suggest that the introduction of the substituents enhances the $K$ P values.

The $n$ values were determined as $1.52-1.94 \times 10^{-5}$ mol g-1, which corresponds to $43-55 \%$ of the amino end group content $\left(3.53 \times 10^{-5} \mathrm{eq}^{-1}\right)$. This suggests that two positively charged amino end groups bind with one dye molecule: 1:1 interaction occurs between a protonated amino end group and a sulfonate group in the dyes [1].

The intrinsic binding constants for the Langmuir type sorption, $K_{\mathrm{L}}$, were influenced by the introduction of the substituents into the dye molecule. In particular, the replacement of the trifluoromethyl group enhanced the $K_{\mathrm{L}}$ values significantly. This result is similar to that for the derivatives of AS which has one sulfonate group [4]. The effects of the trifluoromethyl group might be explained as follows. The introduction of the trifluoromethyl or methyl group changes the electronic structure of the dyes: the charge density of the sulfonate anions changes. As $K_{\mathrm{L}}$ implies the electrostatic binding constant of the sulfonate anions with the positively charged amino end groups in the nylon 6 film, the charge density of the sulfonate anions affects the $K_{\mathrm{L}}$ 
values. In addition, the replacement of the trifluoromethyl or methyl group influences the hydration around the dyes. The number of dehydrated water molecule play an important role in the binding process, so that the change of the hydration also influences the $K_{\mathrm{L}}$ values.

For further discussion, thermodynamic parameters, enthalpy change, $\Delta H_{\mathrm{L}}$, and entropy change, $\Delta S_{\mathrm{L}}$, were determined from the temperature dependence (Table 5). The introduction of the trifluoromethyl group made the binding process more exothermic and less entropic, whereas for the methyl group the opposite behavior was observed. This result reflects the electron-attracting and releasing properties of the substituents. Furthermore, in the case of the dyes containing one sulfonate, the thermodynamic parameters were -45 to $-21 \mathrm{~kJ} \mathrm{~mol}^{-1}$ for $\Delta H_{\mathrm{L}}$ and -9 to $60 \mathrm{~J} \mathrm{~mol}^{-1} \mathrm{~K}^{-1}$ for $\Delta S_{\mathrm{L}}$ [4], while those for the dyes having two sulfonate groups were -37.6 to $2.3 \mathrm{~kJ} \mathrm{~mol}^{-1}$ and 24 to $131 \mathrm{~J} \mathrm{~mol}^{-1} \mathrm{~K}^{-1}$, respectively. This demonstrates that the increase in the number of sulfonate groups amplifies the effects of the trifluoromethyl and methyl group.

Table 5 Thermodynamic Parameters for the Langmuir Type Sorption

\begin{tabular}{lrc}
\hline & $\Delta H_{\mathrm{L}} / \mathrm{kJ} \mathrm{mol}^{-1}$ & $\Delta S_{\mathrm{L}} / \mathrm{J} \mathrm{mol}^{-1} \mathrm{~K}^{-1}$ \\
\hline AR & $-16.5 \pm 1.7$ & $77 \pm 5$ \\
o-FTR & $-24.7 \pm 1.7$ & $62 \pm 5$ \\
m-FTR & $-37.6 \pm 1.6$ & $24 \pm 5$ \\
p-FTR & $-27.6 \pm 1.4$ & $53 \pm 4$ \\
o.TR & $2.3 \pm 0.5$ & $131 \pm 2$ \\
m.TR & $-6.4 \pm 1.7$ & $111 \pm 5$ \\
p-TR & $-0.6 \pm 1.7$ & $127 \pm 5$ \\
\hline
\end{tabular}

To make sure that the thermodynamic compensation relationship $[13,14]$ is operative in the Langmuir type sorption of the dyes by the nylon 6 film, $\Delta H_{\mathrm{L}}$ is plotted against $\Delta S_{\mathrm{L}}$ (Fig. 5). The plot of $\Delta H_{\mathrm{L}}$ against $\Delta S_{\mathrm{L}}$ lays on a straight line for all the dyes, giving $334 \mathrm{~K}$ as compensation temperature. This suggests that all the dyes bind with the nylon 6 film in a similar mode: electrostatic interaction between the anionic groups of the dyes and the protonated amino end groups of the nylon 6 film is dominant and hydrophobic interaction

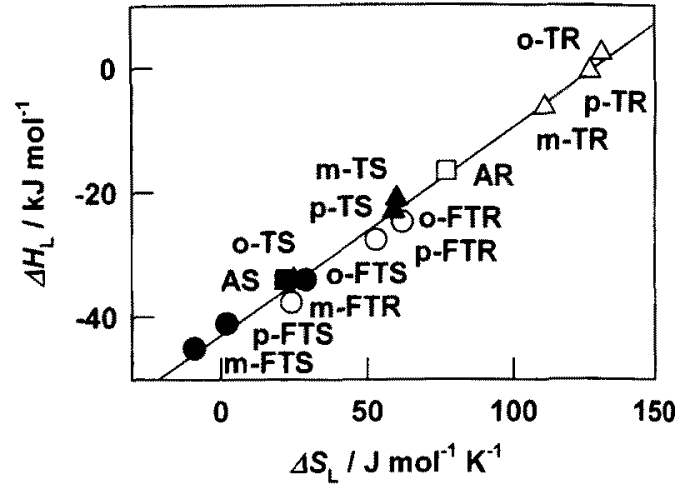

Fig. 5 Compensation relationship between $\Delta H_{\mathrm{L}}$ and $\Delta S_{\mathrm{t}}$.

may be accompanied. The hydrophobicity is one of the most important factor in dye sorption by nylon [6-8].

From the above results, it is concluded that not only the introduction of a trifluoromethyl and methyl group but also the number of sulfonate groups greatly influences the sorption behavior of the dyes by the nylon 6 film.

\section{Acknowledgement}

This work was supported by Grant-in-Aid for COE Research (10CE2003) by the Ministry of Education, Science, Sports and Culture.

\section{References}

1. T. Tak, J. Komiyama, and T. Iijima, Sen'i Gakkaishi, 35, T-486 (1979)

2. V. S. Praptowidodo, K. Hamada, and T. Iijima, Angew. Makromol. Chem., 144, 159 (1986).

3. K. Hamada, T. Mori, and M. Mitsuishi, Sen"i Gakkaishi, 47, 481 (1991).

4. K. Hamada, S. Takemae, and M. Mitsuishi, Sen'i Gakkaishi, 47, 664 (1991).

5. J. Qian, K. Hamada, and M. Mitsuishi, Dyes Pigm., 21, 255 (1993).

6. P. Savarino, E. Barni, G. Viscardi, R. Carpignano, and U. Anselmi, Ann. Chim. (Rome) ,78, 179 (1988).

7. P. Savarino, G. Viscardi, R. Carpignano, and E. Barni, J. Chem. Tech. Biotechnol., 51, 243(1991).

8. P. Savarino, G. Viscardi, R. Carpignano, and E. Barni, Colloids Surf., 35, 251 (1989).

9. K. Hamada and T. Iijima, Dyes Pigm., 16, 253(1991). 
10. K. Hamada, S. Take, T. Iijima, and S. Amiya, $J$. Chem. Soc., Faraday Trans. 1, 82, 3141 (1986).

11. K. Hamada, S. Take, and T. Iijima, Dyes Pigm., 11, 191(1989).

12. K. Hamada, K. Satomura, and M. Mitsuishi, Dyes Pigm., 31, 205(1996).
13. J. E. Leffler and E. Grundwald Eds., "Rates and Equilibria of Organic Reactions", John Wiley, New York (1963).

14. R. Lumry and S. Rajender, Biopolymers, 9, 1125 (1970). 